\title{
DEtection of ProxImal Coronary stenosis in the work-up for Transcatheter aortic valve implantation using CTA (from the DEPICT CTA collaboration)
}

\author{
Thomas P. W. van den Boogert ${ }^{1}$ • Bimmer E. P. M. Claessen ${ }^{2} \cdot$ Maksymilian P. Opolski $^{3} \cdot$ Won-Keun Kim ${ }^{3}$. \\ Ashraf Hamdan ${ }^{4}$. Daniele Andreini ${ }^{5}$. Francesca Pugliese ${ }^{6}$. Helge Möllmann ${ }^{7}$. Ronak Delewi ${ }^{1}$. Jan Baan ${ }^{1}$. \\ M. Marije Vis ${ }^{1} \cdot$ Adrienne van Randen $^{9}$. Joost van Schuppen ${ }^{9} \cdot$ Jaap Stoker $^{8} \cdot$ José P. Henriques $^{1} \cdot$ R. Nils Planken ${ }^{9}$
}

Received: 18 January 2021 / Revised: 12 May 2021 / Accepted: 25 May 2021 / Published online: 16 June 2021

(C) The Author(s) 2021

\begin{abstract}
Objectives Computed tomography angiography (CTA) is performed routinely in the work-up for transcatheter aortic valve implantation (TAVI), and could potentially replace invasive coronary angiography (ICA) to rule out left main (LM) and proximal coronary stenosis. The objectives were to assess the diagnostic yield and accuracy of pre-TAVI CTA to detect LM and proximal coronary stenosis of $\geq 50 \%$ and $\geq 70 \%$ diameter stenosis (DS).

Methods The DEPICT CTA database consists of individual patient data from four studies with a retrospective design that analyzed the diagnostic accuracy of pre-TAVI CTA to detect coronary stenosis, as compared with ICA. Pooled data were used to assess diagnostic accuracy to detect coronary stenosis in the left main and the three proximal coronary segments on a perpatient and a per-segment level. We included 1060 patients (mean age: 81.5 years, $42.7 \%$ male).

Results On ICA, the prevalence of proximal stenosis was $29.0 \%(\geq 50 \%$ DS) and $15.7 \%(\geq 70 \%$ DS). Pre-TAVI CTA ruled out $\geq 50 \%$ DS in $51.6 \%$ of patients with a sensitivity of $96.4 \%$, specificity of $71.2 \%$, PPV of $57.7 \%$, and NPV of $98.0 \%$. For $\geq 70 \%$ DS, pre-TAVI CTA ruled out stenosis in $70.0 \%$ of patients with a sensitivity of $96.7 \%$, specificity of $87.5 \%$, PPV of $66.9 \%$, and NPV of $99.0 \%$.

Conclusion CTA provides high diagnostic accuracy to rule out LM and proximal coronary stenosis in patients undergoing work-up for TAVI. Clinical application of CTA as a gatekeeper for ICA would reduce the need for ICA in 52\% or 70\% of patients, using a threshold of $\geq 50 \%$ or $\geq 70 \%$ DS, respectively.

Key Points

- Clinical application of CTA as a gatekeeper for ICA would reduce the need for ICA in $52 \%$ or $70 \%$ of TAVI patients, using a threshold of $\geq 50 \%$ or $\geq 70 \%$ diameter stenosis.
\end{abstract}

R. Nils Planken

r.n.planken@amsterdamumc.nl

1 Department of Clinical and Experimental Cardiology, Amsterdam Cardiovascular Sciences, Amsterdam UMC, University of Amsterdam, Amsterdam, The Netherlands

2 Department of Cardiology, Noordwest Ziekenhuisgroep, Alkmaar, The Netherlands

3 Department of Interventional Cardiology and Angiology, National Institute of Cardiology, Warsaw, Poland

4 Department of Cardiology, Rabin Medical Center, Sackler Faculty of Medicine, Tel-Aviv University, Tel-Aviv, Israel

5 Department of Cardiology and Radiology, Centro Cardiologico Monzino IRCCS, University of Milan, Milan, Italy
6 NIHR Cardiovascular Biomedical Research Unit at Barts, William Harvey Research Institute, Barts and The London School of Medicine and Dentistry, Queen Mary University of London \& Department of Cardiology, Barts Health NHS Trust, London, UK

7 Department of Cardiology, St. Johannes-Hospital Dortmund, Dortmund, Germany

8 Department of Radiology and Nuclear Medicine, Amsterdam Gastroenterology Endocrinology Metabolism, Amsterdam UMC, University of Amsterdam, Amsterdam, The Netherlands

9 Department of Radiology and Nuclear Medicine, Amsterdam Cardiovascular Sciences, Amsterdam UMC, University of Amsterdam, Meibergdreef 9, 1105, AZ Amsterdam, The Netherlands 
- The diagnostic accuracy of CTA to exclude proximal coronary stenosis in these patients is high, with a sensitivity of $96.4 \%$ and NPV of $98.0 \%$ for a threshold of $\geq 50 \%$, and a sensitivity of $96.7 \%$ and NPV of $99.0 \%$ for a threshold of $\geq 70 \%$ diameter stenosis.

- Atrial fibrillation and heart rate did not significantly affect sensitivity and NPV. However, a heart rate of $<70 \mathrm{~b} / \mathrm{min}$ during CTA was associated with a significantly improved specificity and PPV.

Keywords Coronary artery disease - Aortic valve stenosis - Computed tomography angiography $\cdot$ Diagnostic accuracy · Transcatheter aortic valve replacement

$\begin{array}{ll}\text { Abbreviations } & \\ \text { BMI } & \text { Body mass index } \\ \text { CABG } & \text { Coronary artery bypass grafting } \\ \text { CAD } & \text { Coronary artery disease } \\ \text { CT } & \text { Computed tomography } \\ \text { CTA } & \text { Computed tomography angiography } \\ \text { CX } & \text { Circumflex } \\ \text { DS } & \text { Diameter stenosis } \\ \text { ICA } & \text { Coronary angiography } \\ \text { LAD } & \text { Left anterior descending } \\ \text { LM } & \text { Left main } \\ \text { MDCT } & \text { Multidetector computed tomography } \\ \text { neg-LR } & \text { Negative likelihood ratio } \\ \text { NPV } & \text { Negative predictive value } \\ \text { PCI } & \text { Percutaneous coronary intervention } \\ \text { pos-LR } & \text { Positive likelihood ration } \\ \text { PPV } & \text { Positive predictive value } \\ \text { QUADAS-2 } & \text { Quality Assessment of Studies of } \\ & \text { Diagnostic Accuracy Included } \\ & \text { in Systematic Reviews-2 } \\ \text { RCA } & \text { Right coronary artery } \\ \text { SD } & \text { Standard deviation } \\ \text { TAVI } & \text { Transcatheter aortic valve implantation }\end{array}$

\section{Introduction}

Coronary artery disease (CAD) is a common concurrent condition in patients with aortic valve stenosis undergoing transcatheter aortic valve implantation (TAVI). Pre-procedural screening for CAD is recommended by the current TAVI guidelines and is usually performed with invasive coronary angiography (ICA) [1-3]. However, pre-procedural revascularization with percutaneous coronary intervention (PCI) is only recommended to consider in patients with coronary stenosis of more than $70 \%$ diameter stenosis (DS) in proximal coronary artery segments [3]. Consequently, the majority of patients undergo ICA solely for the exclusion of severe proximal coronary stenosis. This invasive test is associated with a risk of complications and high consumption of healthcare resources. An alternative noninvasive diagnostic test to rule out obstructive $\mathrm{CAD}$ is computed tomography angiography (CTA), which is routinely performed in the pre-TAVI work-up for appropriate prosthesis sizing and evaluation of access routes.

A previous analysis showed that obstructive CAD on CTA could be excluded in only $37 \%$ of TAVI patients if all coronary segments were evaluated [4]. Considering that only proximal coronary arteries need evaluation according to the current guidelines, we hypothesized that CTA could exclude a higher percentage of clinically relevant proximal stenosis. Therefore, we collected individual patient data from 1060 subjects from studies that investigated the diagnostic accuracy of CTA as compared to ICA to detect obstructive coronary stenosis in patients who were evaluated for TAVI. In this data set, we assessed the diagnostic yield and accuracy of pre-TAVI CTA to detect left main (LM) and proximal coronary stenosis.

\section{Materials and methods}

\section{Study design, patient population, and study selection}

This collaborative study contains patients who underwent both CTCA and ICA in the diagnostic work-up for TAVI. The patient population was selected through a literature search in OVID MEDLINE (including Epub Ahead of Print, InProcess \& Other Non-Indexed Citations) and OVID EMBASE from January 1, 1990, to October 1, 2019. We searched for the concepts of TAVI and CTA, using controlled terms like MesH and text words. No language, date, or other restrictions were applied. The reference lists and citing articles of the identified relevant papers were cross-checked in Web of Science. Studies were considered for inclusion if they complied with the following requirements: original studies reporting on the diagnostic accuracy of CTA to detect coronary stenosis, used ICA as reference standard, reported on patients in the work-up for TAVI. Eight studies matched the criteria and the authors were approached for collaboration [5-12]. The authors of four studies could accommodate the data for a per-segment analysis [5-8]. All included patients have been previously reported [5-8]. These prior articles reported on the diagnostic accuracy of CTA, as compared to ICA, to detect obstructive CAD in all coronary segments whereas in this manuscript we report on the diagnostic accuracy of CTA, as compared to ICA, to detect obstructive CAD 
in the proximal coronary segments only. All patients provided written informed consent according to the policy of each participating hospital. The methodological quality was assessed using the modified Quality Assessment of Studies of Diagnostic Accuracy Included in Systematic Reviews-2 (QUADAS-2) criteria [13].

\section{Data collection}

The data sets included patient characteristics regarding age, sex, body mass index (BMI), and the heart rate during CT scan. Information about comorbidities included the presence of diabetes mellitus, atrial fibrillation, hypercholesterolemia, peripheral arterial disease, hypertension, smoking, and a history of CAD, PCI, and coronary artery bypass grafting. Technical CT scanner information included scanner type, number of detector rows, number of slices, detector width, CT scanner rotation time, scan protocol and settings (tube voltage and tube current), contrast agent type (concentration) and volume, dose length product, and nitroglycerin use. The data sets included stenosis grading for both CTA and ICA in the left main (LM) and the proximal segments of the right coronary artery (RCA), left anterior descending (LAD) artery, and circumflex (CX), according to the American Heart Association or Society of Cardiovascular Computed Tomography classification. Both use the same definition for the proximal segments. Proximal RCA is defined as the ostium to one-half the distance to the acute margin of the heart, the LM as the ostium to the bifurcation of the LAD artery and $\mathrm{CX}$, the proximal LAD artery from the end of the LM to the first diagonal, and the proximal CX as the end of LM to the origin of the obtuse marginal. For stenosis grading, all studies used a cut-off value of $\geq 50 \%$ DS to determine the presence of obstructive CAD. Three out of four studies also reported an additional cut-off value of $\geq 70 \%$ DS [6-8].

\section{CTA acquisition}

CTA acquisition in the included studies was performed using the following CT scanners: LightSpeed VCT XTe Scanner (GE Healthcare) [7], Somatom Definition (Siemens) [5], Somatom Definition Flash (Siemens) [8], Philips iCT (Philips Healthcare) [6]. All studies used a retrospective ECG-gated low-pitch spiral protocol, with CT scanner setting of $80-140$ kilovolts $(\mathrm{kV})$ and $185-600 \mathrm{~mA}$ per rotation (Table 1). The studies reported different contrast injection protocols with a mean total volume of $109.5 \mathrm{ml}$, ranging between 50 and $170 \mathrm{ml}$. The iodine concentration in the contrast medium varied between 300 and $400 \mathrm{mg} \mathrm{I} / \mathrm{ml}$, with a majority of $44.8 \%$ having $370 \mathrm{mg} \mathrm{I} / \mathrm{ml}$ [5]. This translated to a mean total iodine load of $40.8 \mathrm{~g}$ iodine with standard deviation of $10.4 \mathrm{~g}$ iodine. The mean dose length product of the TAVI
Table 1 Baseline table and CT characteristics table

\begin{tabular}{|c|c|}
\hline \multicolumn{2}{|l|}{ Patient demographics } \\
\hline Number of patients, $\mathrm{n}$ & 1060 \\
\hline Age (years, SD) & $81.7 \pm 6.6$ \\
\hline Male gender, n (\%) & $545(51.4)$ \\
\hline BMI, $\left(\mathrm{kg} / \mathrm{m}^{2}, \mathrm{SD}\right)$ & $26.8 \pm 4.9$ \\
\hline Diabetes mellitus, n (\%) & $224(21.3)$ \\
\hline Atrial fibrillation, n (\%) & $159(15.5)$ \\
\hline Hyperlipidemia, n (\%) & $543(51.8)$ \\
\hline Hypertension, n (\%) & $886(84.0)$ \\
\hline Heart rate during CTA, $n$ (SD) & $69.3 \pm 12.8$ \\
\hline History of PCI, n (\%) & $300(29.8)$ \\
\hline History of CABG, n (\%) & $162(16.1)$ \\
\hline \multicolumn{2}{|l|}{ CT details and settings } \\
\hline \multicolumn{2}{|l|}{ Detector rows [width], n (\%) } \\
\hline $40[24]$ & $475(44.8)$ \\
\hline $64[38.4]$ & $140(13.2)$ \\
\hline $64[40]$ & $330(31.1)$ \\
\hline $256[160]$ & $115(10.8)$ \\
\hline \multicolumn{2}{|l|}{ Tube voltage (\%) } \\
\hline 80 & $91(8.6)$ \\
\hline 100 & $394(37.2)$ \\
\hline 120 & $573(54.1)$ \\
\hline 140 & $1(0.1)$ \\
\hline \multicolumn{2}{|l|}{ Tube current (\%) } \\
\hline $300-400$ & $615(58.1)$ \\
\hline $400-600$ & $247(23.3)$ \\
\hline $600-800$ & $82(7.7)$ \\
\hline Contrast volume (SD) & $109.5 \pm 20.8$ \\
\hline \multicolumn{2}{|l|}{ Contrast concentration (\%) } \\
\hline 300 & $140(13.2)$ \\
\hline 350 & $115(10.8)$ \\
\hline 370 & $475(44.8)$ \\
\hline 400 & $330(31.1)$ \\
\hline
\end{tabular}

Baseline characteristics and CT details and settings are listed for the patients included in the analysis. Abbreviations: $B M I$, body mass index; $C T A$, computed tomography angiography; $P C I$, percutaneous coronary intervention; $C A B G$, coronary artery bypass graft

CTA was $1910 \pm 616.3 \mathrm{mGy} * \mathrm{~cm}$ and included all CTA sequences used for TAVI planning.

\section{Invasive coronary angiography acquisition}

In the included studies, ICA served as the reference standard and was performed by experienced readers who were blinded for the CTA results. Three out of four studies used off-line quantitative coronary angiography for stenosis assessment and evaluated segments in at least 2 orthogonal projections [6-8]. One study evaluated coronary stenosis by visual assessment [5]. 


\section{Objectives}

The primary objective was to assess the diagnostic yield and accuracy of pre-TAVI CTA to detect coronary stenosis ( $\geq 50 \%$ DS and $\geq 70 \%$ DS) in the LM and proximal coronary segments on a per-patient and a per-segment level. The secondary objective was to perform a subgroup analysis of the individual studies and to assess the influence of atrial fibrillation and heart rate on the diagnostic accuracy of CTA.

\section{Statistical analysis}

Data analysis was performed using the statistical software $\mathrm{R}$ version 3.5.1 (R Foundation for Statistical Computing). Continuous variables were presented as means with standard deviations (SD). The distribution of continuous variables was tested with the Shapiro-Wilk test. Categorical variables were presented as frequencies and percentages. The prevalence of proximal CAD was based on ICA. Diagnostic accuracy of pre-TAVI CTA, as compared to pre-TAVI ICA, was defined as the sensitivity, specificity, positive predictive value (PPV), negative predictive value (NPV), positive likelihood ration (pos-LR), and negative likelihood ratio (neg-LR). The analysis was performed on a per-patient and per-segment level. All non-diagnostic segments were labelled as if there was a coronary stenosis. Diagnostic yield was defined as the sum of the negatives (true negatives and false negatives) and presented for both the total of patients and for a subgroup without patients with $\mathrm{CABG}$. For subgroup analysis, we defined subgroups of patients with and without atrial fibrillation as well as subgroups with a heart rate $<70$ and $\geq 70$ beats $/ \mathrm{min}$. The diagnostic accuracy in these subgroups was assessed on a perpatient level for stenosis $\geq 50 \%$ DS. Diagnostic accuracy measures were compared using the Pearson's chi-squared test statistic.

\section{Results}

\section{Baseline characteristics}

Patient selection is summarized in Fig. 1 and baseline and CT scan characteristics are listed in Table 1 . The combined studies included 1060 patients with a mean age of $81.7 \pm 6.6$ years and $42.7 \%$ of patients were male. The mean BMI was $26.8 \pm$ $4.9 \mathrm{~kg} / \mathrm{m}^{2}$, diabetes mellitus was present in $28.3 \%$ of our study population, and $24.7 \%$ had atrial fibrillation. A total of 300 patients $(29.8 \%)$ had prior percutaneous coronary intervention (PCI) and $16.1 \%$ had previous coronary artery bypass grafting. Mean heart rate during pre-TAVI CTA was 69.3 beats/min and varied between $61 / \mathrm{min}$ and $74 / \mathrm{min}$ in the included studies. Methodological quality assessment of included studies by QUADAS-2 is discussed in supplementary material (supplemental text and supplemental table 1 and supplemental figure 1).

\section{Prevalence of obstructive proximal CAD at ICA}

Using ICA as a reference standard, proximal stenosis of $\geq$ $50 \%$ DS was present in 307 of 1060 patients (29.0\%), with coronary stenosis located in the proximal RCA $(n=193$, $18.2 \%)$, the LM coronary artery $(\mathrm{n}=47,4.4 \%)$, the proximal LAD artery $(n=162,15.3 \%)$, and the proximal CX $(n=120$, $11.3 \%$ ). The prevalence differed between the populations included in the individual studies. The lowest prevalence was $15.7 \%$ in the study of Rossi et al [8], followed by $18.8 \%$ in the study of Andreini et al [5], 32.2\% in the study of Hamden et al [7], and 39.2\% in the study of Opolski et al [6].

In a subgroup of 585 patients, data was also available for an additional coronary stenosis threshold $\geq 70 \%$ DS. Proximal coronary stenosis $\geq 70 \%$ DS was present in 92 of 585 patients $(15.7 \%)$, with coronary stenosis located in the proximal RCA $(\mathrm{n}=57,9.7 \%)$, the LM $(\mathrm{n}=4,0.7 \%)$, the proximal LAD artery $(n=46,7.9 \%)$, and the proximal CX $(n=27,4.6 \%)$.

\section{Accuracy of CTA for the detection of obstructive proximal CAD}

\section{Stenosis $\geq \mathbf{5 0 \%}$ DS}

The diagnostic accuracy for the detection of proximal $\geq 50 \%$ DS is listed in Table 2 and summarized in Fig. 2. The diagnostic yield of CTA by ruling out proximal $\geq 50 \%$ DS was $51.6 \%$ (547 patients, 536 true negatives +11 false negatives). The consistency rate of CTA and ICA was $78.5 \%$. The sensitivity and NPV of CTA to detect $\geq 50 \%$ DS were $96.4 \%$ and $98.0 \%$, respectively. The specificity and the PPV were $71.2 \%$ and $57.7 \%$, respectively. In the $513(48.4 \%)$ patients in whom coronary stenosis was not ruled out, CTA showed proximal $\geq$ $50 \%$ DS in 315 patients (29.7\%) and non-evaluable proximal segments in 198 patients (18.7\%). All corresponding standard deviations and positive and negative likelihood ratios (LR) are listed in Table 2, together with the location of coronary stenosis and non-evaluable segments. In the 898 patients without CABG, the diagnostic yield was $56.6 \%$ (508, 499 true negatives +9 false negatives). On a per-segment level (including the non-evaluable segments), CTA correctly classified $89.7 \%$ of all proximal segments. CTA correctly identified 460 out of 506 proximal coronary stenosis $\geq 50 \%$ DS, resulting in a sensitivity of $90.9 \%$. Of the 3733 segments without obstructive stenosis, CTA correctly ruled out obstructive stenosis in 3342 segments, resulting in a specificity of $89.5 \%$. The PPV was $54.1 \%$ and the NPV was $98.6 \%$. 
Fig. 1 Flowchart of patient selection in the included patient population. A total of 1060 out of 1258 patients who underwent both CTA and ICA in the workup for TAVI were included in the final analysis of the individual studies. Reasons for exclusion of patients are listed. Abbreviations: ICA, invasive coronary angiography; CTA, computed tomography angiography; TAVI, transcatheter aortic valve implantation

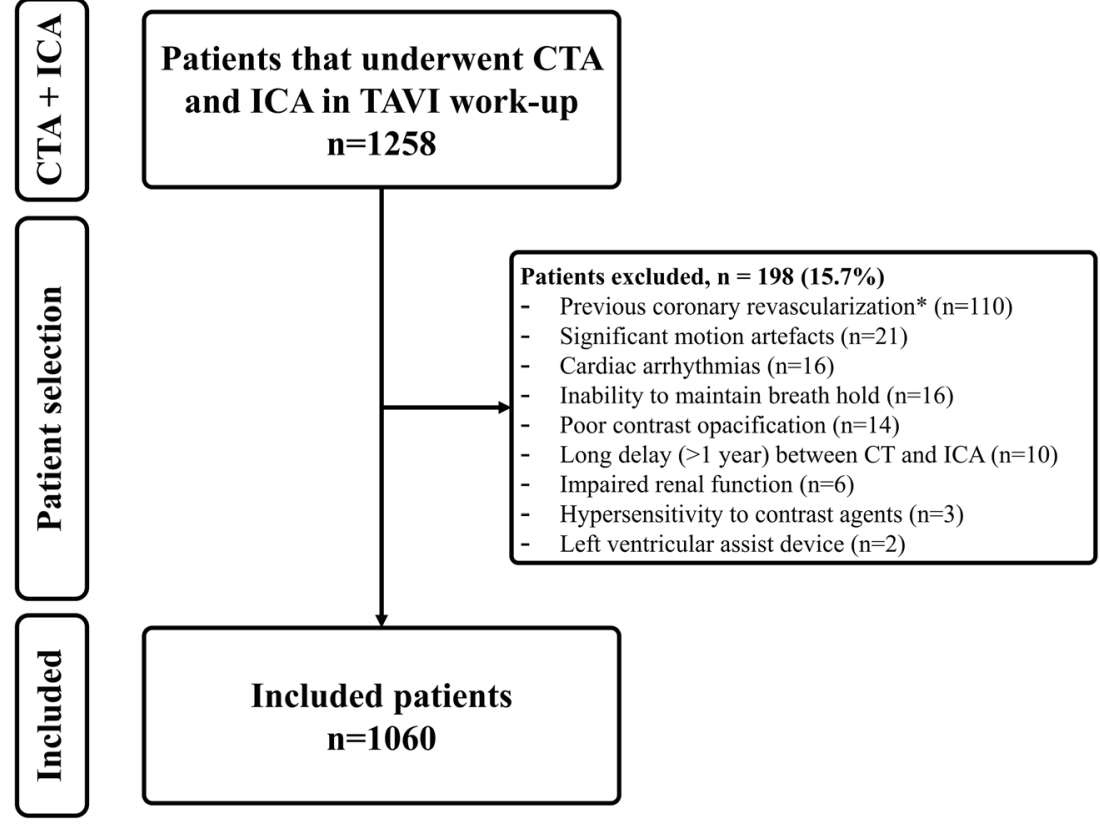

\section{Stenosis $\geq 70 \%$ DS}

Data to calculate the diagnostic accuracy for the detection of proximal $\geq 70 \%$ DS was available in 585 patients (Table 3 , Fig. 2). The diagnostic yield of CTA to rule out proximal $\geq$ $70 \%$ DS was $70.1 \%$ (410 patients, 406 true negatives +4 false negatives). The consistency rate of CTA and ICA was $89.4 \%$. The sensitivity and NPV of CTA to detect $\geq 70 \%$ DS were $96.7 \%$ and $99.0 \%$, respectively. The specificity and the PPV were $87.5 \%$ and $66.9 \%$, respectively. In the 496 patients without CABG, the diagnostic yield was $72.2 \%$ (358, 356 true negatives +2 false negatives).

On a per-segment level (including the non-evaluable segments), CTA correctly classified $98.1 \%$ of segments, resulting in a sensitivity of $87.8 \%$, specificity of $98.7 \%$, PPV of $80.0 \%$, and NPV of $99.3 \%$, respectively. In 42 out of 2257 evaluable segments, CTA misclassified a coronary stenosis with a threshold of $\geq 70 \%$ DS. These misclassifications were the result of overestimation of the stenosis in 27 cases (false positives) and underestimation of the stenosis in 15 cases (false negatives).

\section{The influence of atrial fibrillation and heart rate}

In the subgroup of 159 patients with atrial fibrillation, perpatient evaluation with CTA correctly identified $79.2 \%$ of patients (vs. $78.5 \%$ of correctly identified patients in the total cohort), with a sensitivity of $100 \%$, specificity of $72.0 \%$, PPV of $55.4 \%$, and NPV of $99.4 \%$. Compared to the patients with atrial fibrillation, CTA correctly identified proximal $\geq 50 \%$ DS in $74.8 \%$ of patients without atrial fibrillation $(p=0.27)$. There was also no significant difference in the diagnostic accuracy measures of the patients without atrial fibrillation (sensitivity of $95.7 \%$, specificity of $70.2 \%$, PPV of $56.1 \%$, and NPV of $97.6 \%$ ).

In the subgroup of 552 patients with a heart rate $<70 \mathrm{~b} / \mathrm{min}$, per-patient evaluation with CTA correctly identified $84.6 \%$ of patients, with a sensitivity of $96.3 \%$, a specificity of $79.6 \%$, PPV of $66.7 \%$, and NPV of $98.1 \%$. In the subgroup with a heart rate of $\geq 70 \mathrm{~b} / \mathrm{min}$, CTA correctly identified significantly less patients $(71.5 \%, p \leq 0.001)$. There was no significant difference in the sensitivity and NPV (sensitivity of $96.5 \%$ and NPV of $97.8 \%$ ). However, specificity and PPV were significantly lower than in the patients with heart rate $<70 \mathrm{~b} / \mathrm{min}$ (specificity of $61.8 \%, p \leq 0.001$ and PPV of $49.6 \%, p \leq 0.001$ )

\section{Discussion}

Our findings demonstrate that assessment of proximal stenosis in the TAVI work-up by CTA can be used as a gatekeeper for ICA in routine clinical practice. The highly sensitive CTA is capable to rule out proximal $\geq 50 \%$ DS in $52 \%$ of patients and proximal $\geq 70 \%$ DS in $70 \%$ of patients, hereby substantially reducing the need for ICA in the work-up for TAVI. However, the relative low specificity and PPV may limit the clinical utility and applicability of CTA to guide coronary revascularization in the TAVI candidates.

Pre-TAVI screening for CAD is required to estimate baseline procedural risk and indicate if patients need revascularization prior to TAVI. The prevalence of obstructive CAD in all coronary segments ranges from 40 to $70 \%$ according to several large multicenter registries such as the FRANCE 2 and the PARTNER TAVI registries [2]. However, the prognostic role of CAD in patients undergoing TAVI remains 


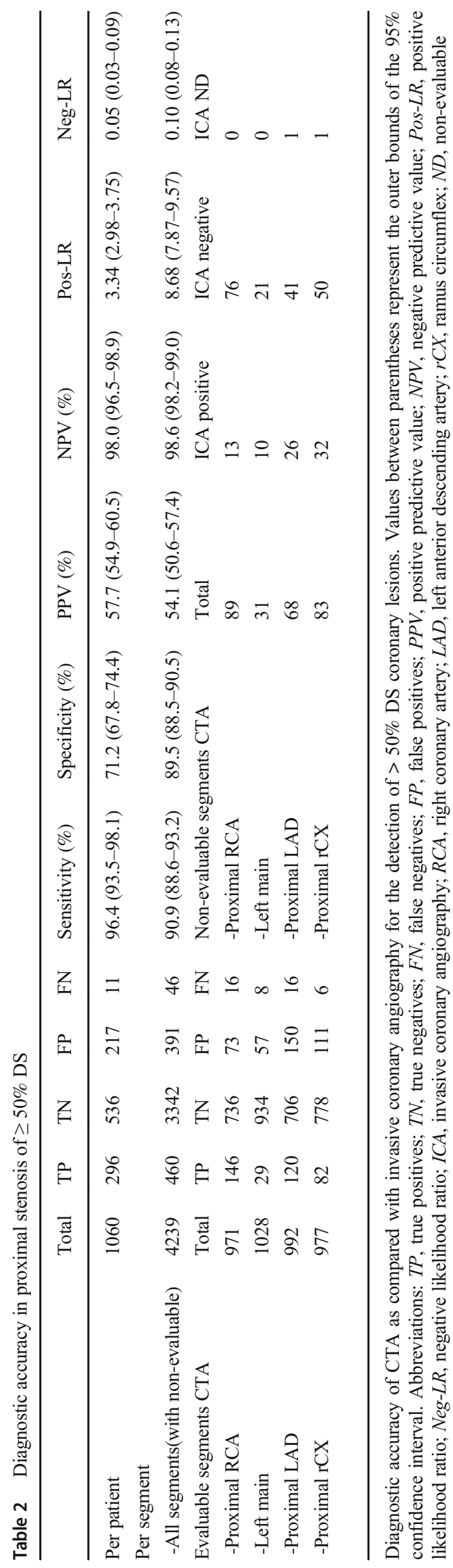

unknown and the most recent guidelines recommend that revascularization should only be considered in proximal coronary stenosis $\geq 70 \%$ DS [3]. If only the proximal coronary segments are evaluated, only a minority of patients would qualify for obstructive CAD. In our cohort, proximal obstructive CAD was only prevalent in $29.0 \%$ of patients on the basis of a $\geq 50 \%$ DS cut-off value and $15.7 \%$ of patients on the basis of $a \geq 70 \%$ DS cut-off value. To our knowledge, the only other study that reported a prevalence of proximal coronary artery stenosis $\geq 50 \%$ DS in TAVI patients, found a prevalence of $17 \%$ [14]. That study excluded patients with a history of acute coronary syndrome or previous coronary revascularization, causing bias and lowering the prevalence of obstructive proximal CAD.

Between the studies that were included in this collaborative study, the prevalence of obstructive proximal CAD ranged between 15.7 and $39.2 \%$. The study by Rossi et al excluded all patients with prior coronary revascularization (a total of 110 patients, $44 \%$ of total), resulting in the lowest prevalence of obstructive proximal CAD (15.7\%). On the contrary, the study by Opolski et al reported the lowest percentage of excluded patients (6.9\%) and the highest prevalence of obstructive proximal CAD (39.2\%)[5]. These differences could have led to the differences in overall diagnostic accuracy. Besides prevalence, the difference in diagnostic accuracy of CTA can be explained by the differences in CT scanner technology. The studies with the least advanced CT scanners had the lowest overall diagnostic accuracy [5, 7]. Conversely, studies with the most advanced CT scanners had the highest overall diagnostic accuracy $[6,8]$.

We tested the influence of previous or current atrial fibrillation and heart rate during $\mathrm{CT}$ acquisition on the diagnostic accuracy of CTA. The diagnostic accuracy of CTA in the patients with atrial fibrillation was comparable to patients in the overall cohort and should therefore not be considered a limitation for clinical implementation. Sensitivity, NPV, and diagnostic yield for ruling out proximal $>50 \%$ DS were not statistically different between patients with a heart rate $>70$ $\mathrm{b} / \mathrm{min}$ versus $<70 \mathrm{~b} / \mathrm{min}$. However, a heart rate of $<70 \mathrm{~b} / \mathrm{min}$ during CTA was associated with a significantly improved specificity and PPV, compared to the patients with heart rate of $\geq 70 \mathrm{~b} / \mathrm{min}$, most likely due to more motion artifacts in the latter group. Two out of four included studies applied heart rate control using negatively chronotropic medication resulting in a mean heart rate of $63.7 \mathrm{~b} / \mathrm{min}$, compared to $73.4 \mathrm{~b} / \mathrm{min}$ in the other studies $[6,7]$. Therefore, heart rate control in TAVI patients could potentially improve diagnostic accuracy of CTA to detect coronary stenosis. Besides heart rate control, the use of nitroglycerin could increase the diagnostic accuracy of CTCA in TAVI patients. However, the risk of decreased blood pressure in these fragile patients with aortic valve pathology is controversial and may raise the need for dedicated monitoring during CTA acquisition. 
Fig. 2 Diagnostic yield and accuracy of CTA to detect proximal coronary stenosis, using a threshold of $\geq 50 \%$ and $70 \%$ diameter stenosis. Abbreviations: ICA, invasive coronary angiography; CTA, computed tomography angiography; NPV, negative predictive value; PPV, positive predictive value; TAVI, transcatheter aortic valve implantation

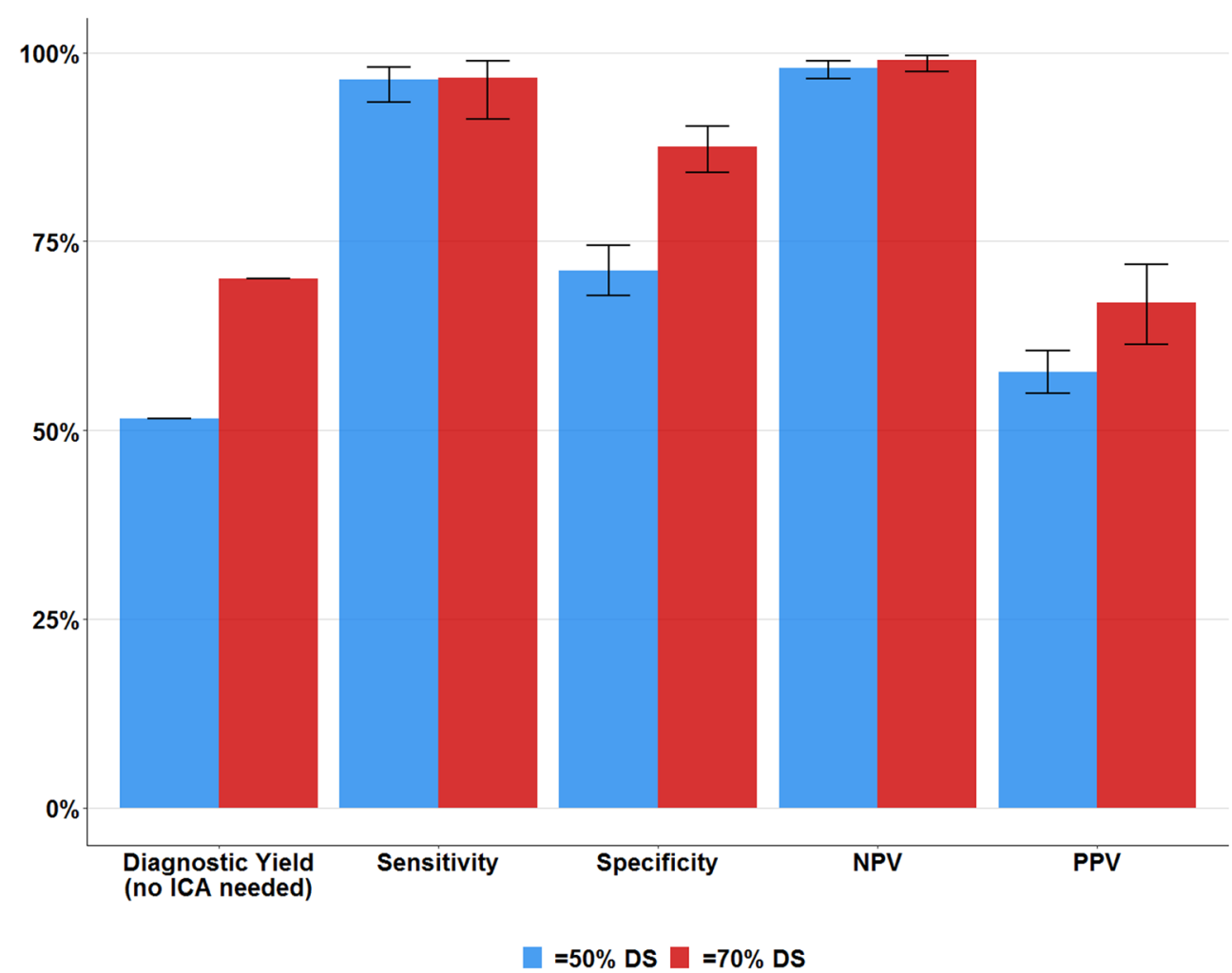

\section{Clinical implications}

Since CTA might be used for both CAD screening and preprocedural TAVI planning, the combined clinical use in the TAVI work-up seems practical. Our results indicate that additional ICA could be avoided in $52 \%$ and $70 \%$ of patients in a relatively frail TAVI population if thresholds of $\geq 50 \%$ DS and $\geq 70 \%$ DS were used, respectively. TAVI patients are generally at increased age and have increased risk of bleeding and of vascular, embolic, and neurological complications [15, 16]. Major vascular complications were registered in $3.6 \%$ of elderly patients undergoing diagnostic ICA [17]. Therefore, avoiding ICA could reduce complications in the TAVI work-up. Reducing the number of diagnostic tests that use nephrotoxic contrast material is desirable in a patient population that is at increased risk of contrast-induced acute kidney injury [18].

In clinical practice, two cut-off values for obstructive coronary stenosis are widely used ( $\geq 50 \%$ DS and $\geq 70 \%$ DS). Currently, there are no studies available investigating the effect of PCI in individual patients with proximal obstructive stenosis $(\geq 70 \% \mathrm{DS}$ ), or patients with functional or hemodynamic significant stenosis. The effect of pre-TAVI PCI in this stenosis has to be elucidated. The preliminary results of the randomized ACTIVATION trial suggest that pre-TAVI revascularization is not linked to changes in 1 year outcomes after TAVI [19]. Until these final results become available, we recommend to maintain a $50 \%$ DS cut-off value as a safety margin and to discuss all patients with proximal coronary stenosis of $\geq 50 \%$ DS in a multidisciplinary heart valve team to determine indications for additional ICA.

\section{Limitations}

This study is an analysis of patient data, generated from four single-center observational studies, which mainly included unselected TAVI patients. One study excluded patients with a history of prior coronary revascularization, potentially influencing the prevalence of CAD. The other studies used inclusion and exclusion criteria that may have resulted in the selection of patients with better image quality CT scans and thus overestimate the diagnostic accuracy (exclusion of patients with cardiac arrhythmias, left ventricular assist device, significant motion artifacts on CTA, or poor contrast opacification). Another limitation of this study is that all CTA scans in the individual studies were acquired with older generation CT scanners. Modern CT scanners would likely have increased the diagnostic yield and accuracy to detect proximal CAD in TAVI patients. Furthermore, all included studies used different contrast injection protocols and different contrast media concentrations. Due to the small number of studies with differences in both CT scanner settings and contrast injection parameters, it is impossible to formulate recommendations on contrast injection protocols or specific contrast media to improve diagnostic accuracy. 


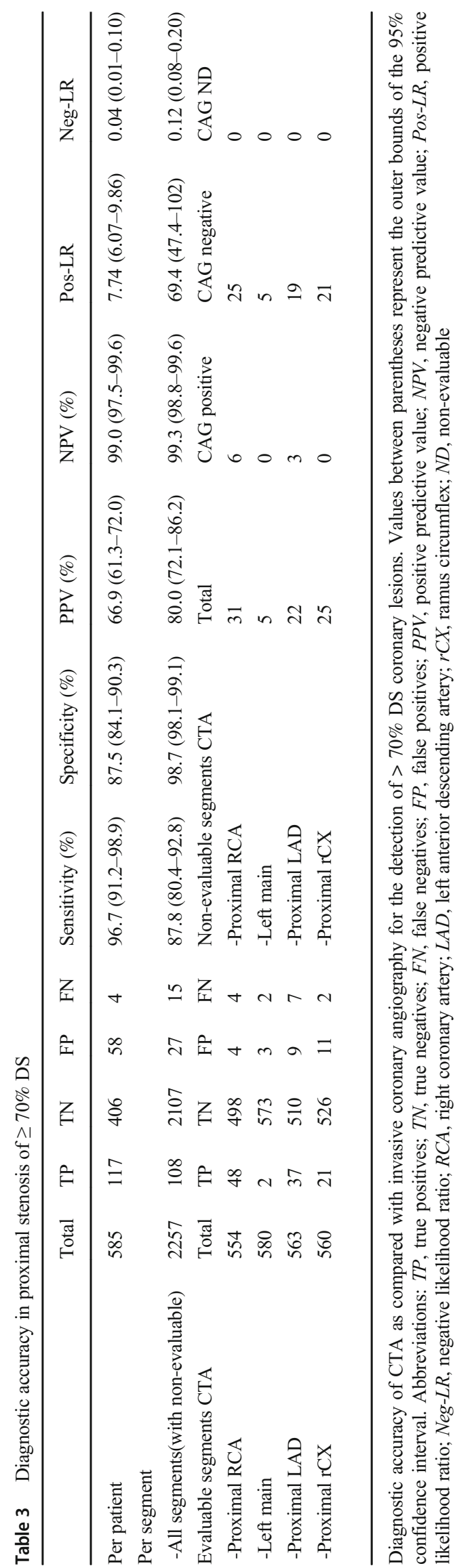

\section{Conclusion}

CTA provides high diagnostic accuracy to rule out LM and proximal coronary stenosis in patients undergoing work-up for TAVI. Clinical application of CTA as a gatekeeper for ICA would reduce the need for ICA in $52 \%$ or $70 \%$ of patients, using a threshold of $\geq 50 \%$ or $\geq 70 \%$ DS, respectively.

Supplementary Information The online version contains supplementary material available at https://doi.org/10.1007/s00330-021-08095-2.

Acknowledgements J. Baan Jr. receives an unrestricted research grant from Edwards Lifesciences and is proctor for Edwards Lifesciences. W. Kim received proctor or speaker fees from Edwards Lifesciences, Boston Scientific, Abbott, and Medtronic. The remaining authors have nothing to disclose.

Funding The authors state that this work has not received any funding.

\section{Declarations}

Guarantor The scientific guarantor of this publication is Nils Planken.

Conflict of interest The authors of this manuscript declare no relationships with any companies whose products or services may be related to the subject matter of the article.

Statistics and biometry One of the authors has significant statistical expertise.

Informed consent Written informed consent was obtained from all subjects (patients) in this study.

Ethical approval Institutional Review Board approval was obtained.

Study subjects or cohorts overlap Some study subjects or cohorts have been previously reported in previous studies, as described in the manuscript. The previous studies described the diagnostic accuracy of CTA, as compared to invasive coronary angiography, to diagnose coronary lesions in all segments of the coronary tree in patients who underwent TAVI. The current study combines the cohorts of the previous studies and investigates the diagnostic accuracy of CTA, as compared to invasive coronary angiography, in the proximal coronary segments only.

The studies that described study subjects, included in the current cohort:

1. Andreini D, Pontone G, Mushtaq S, Bartorelli AL, Ballerini G, Bertella E, et al Diagnostic accuracy of multidetector computed tomography coronary angiography in 325 consecutive patients referred for transcatheter aortic valve replacement. Am Heart $J$ [Internet]. 2014;168(3):332-9. Available from: https://doi.org/10.1016/j.ahj.2014. 04.022

2. Rossi A, De Cecco CN, Kennon SRO, Zou L, Meinel FG, Toscano $\mathrm{W}$, et al CT angiography to evaluate coronary artery disease and revascularization requirement before trans-catheter aortic valve replacement. $J$ Cardiovasc Comput Tomogr. 2017;11(5):338-46.

3. Hamdan A, Wellnhofer E, Konen E, Kelle S, Goitein O, Andrada $\mathrm{B}$, et al Coronary CT angiography for the detection of coronary artery stenosis in patients referred for transcatheter aortic valve replacement. $J$ Cardiovasc Comput Tomogr [Internet]. 2015;9(1):31-41. Available from: https://doi.org/10.1016/j.jcct.2014.11.008 
4. Opolski MP, Christoph WK, Linden A Van, Walther T, Hamm $\mathrm{CW}$, Mo H. Diagnostic accuracy of computed tomography angiography for the detection of coronary artery disease in patients referred for transcatheter aortic valve implantation. 2015;471-80.

\section{Methodology}

- Retrospective

- Diagnostic or prognostic

- Multicenter study

Open Access This article is licensed under a Creative Commons Attribution 4.0 International License, which permits use, sharing, adaptation, distribution and reproduction in any medium or format, as long as you give appropriate credit to the original author(s) and the source, provide a link to the Creative Commons licence, and indicate if changes were made. The images or other third party material in this article are included in the article's Creative Commons licence, unless indicated otherwise in a credit line to the material. If material is not included in the article's Creative Commons licence and your intended use is not permitted by statutory regulation or exceeds the permitted use, you will need to obtain permission directly from the copyright holder. To view a copy of this licence, visit http://creativecommons.org/licenses/by/4.0/.

\section{References}

1. Otto CM, Alexander KP, Calhoon JH et al (2017) 2017 ACC expert consensus decision pathway for transcatheter aortic valve replacement in the management of adults with aortic stenosis. J Am Coll Cardiol 69:1314-1346. https://doi.org/10.1016/j.jacc.2016.12.006

2. Goel SS, Ige M, Tuzcu EM et al (2013) Severe aortic stenosis and coronary artery disease - implications for management in the transcatheter aortic valve replacement era: a comprehensive review. J Am Coll Cardiol 62:1-10. https://doi.org/10.1016/j.jacc.2013.01. 096

3. Baumgartner H, Falk V, Bax JJ et al (2017) 2017 ESC/EACTS guidelines for the management of valvular heart disease. Eur. Heart J 38:2739-2786. https://doi.org/10.1093/eurheartj/ehx391

4. van den Boogert TPW, Vendrik J, Claessen BEPM et al (2018) CTCA for detection of significant coronary artery disease in routine TAVI work-up: a systematic review and meta-analysis. Neth Hear J 26:591-599. https://doi.org/10.1007/s12471-018-1149-6

5. Opolski MP, Kim WK, Liebetrau C et al (2015) Diagnostic accuracy of computed tomography angiography for the detection of coronary artery disease in patients referred for transcatheter aortic valve implantation. Clin Res Cardiol 104(6):471-480. https://doi. org/10.1007/s00392-014-0806-Z

6. Hamdan A, Wellnhofer E, Konen E et al (2015) Coronary CT angiography for the detection of coronary artery stenosis in patients referred fortranscatheter aortic valve replacement. J Cardiovasc Comput Tomogr 9:31-41. https://doi.org/10.1016/j.jcct.2014.11. 008

7. Andreini D, Pontone G, Mushtaq S et al (2014) Diagnostic accuracy of multidetector computed tomography coronary angiography in 325 consecutive patients referred for transcatheter aortic valve replacement. Am Heart J 168:332-339. https://doi.org/10.1016/j.ahj. 2014.04.022
8. Rossi A, De Cecco CN, Kennon SRO et al (2017) CT angiography to evaluate coronary artery disease and revascularization requirement before trans-catheter aortic valve replacement. J Cardiovasc Comput Tomogr 11:338-346. https://doi.org/10.1016/j.jcct.2017. 06.001

9. Harris BS, De Cecco CN, Schoepf UJ et al (2015) Dual-source CT imaging to plan transcatheter aortic valve replacement: accuracy for diagnosis of obstructive coronary artery disease. Radiology 275 : 80-88. https://doi.org/10.1148/radiol.14140763

10. Matsumoto S, Yamada Y, Hashimoto M et al (2017) CT imaging before transcatheter aortic valve implantation (TAVI) using variable helical pitch scanning and its diagnostic performance for coronary artery disease. Eur Radiol 27:1963-1970. https://doi.org/10. 1007/s00330-016-4547-4

11. Annoni AD, Andreini D, Pontone G et al (2018) CT angiography prior to TaVI procedure using third-generation scanner with wide volume coverage: feasibility, renal safety and diagnostic accuracy for coronary tree. Br J Radiol 91:1-11. https://doi.org/10.1259/bjr. 20180196

12. Pontone G, Andreini D, Bartorelli AL et al (2011) Feasibility and accuracy of a comprehensive multidetector computed tomography acquisition for patients referred for balloon-expandable transcatheter aortic valve implantation. Am Heart J 161:1106-1113. https:// doi.org/10.1016/j.ahj.2011.03.003

13. Whiting PF, Rutjes AWS, Westwood ME et al (2011) Quadas-2: a revised tool for the quality assessment of diagnostic accuracy studies. Ann Intern Med 155:529-536. https://doi.org/10.7326/00034819-155-8-201110180-00009

14. Strong C, Ferreira A, Teles RC et al (2019) Diagnostic accuracy of computed tomography angiography for the exclusion of coronary artery disease in candidates for transcatheter aortic valve implantation. Sci Rep 9:1-6. https://doi.org/10.1038/s41598-019-56519-3

15. Madhavan MV, Gersh BJ, Alexander KP et al (2018) Coronary artery disease in patients $\geq 80$ years of age. $\mathrm{J}$ Am Coll Cardiol 71: 2015-2040. https://doi.org/10.1016/j.jacc.2017.12.068

16. Gersh BJ, Kronmal RA, Frye RL et al (1983) Coronary arteriography and coronary artery bypass surgery: morbidity and mortality in patients ages 65 years or older. A report from the coronary artery surgery study. Circulation 67:483-491. https://doi.org/10.1161/01. CIR.67.3.483

17. Niebauer J, Sixt S, Zhang F et al (2004) Contemporary outcome of cardiac catheterizations in 1085 consecutive octogenarians. Int J Cardiol 93:225-230. https://doi.org/10.1016/S0167-5273(03) 00216-X

18. Giordana F, D'Ascenzo F, Nijhoff F et al (2014) Meta-analysis of predictors of all-cause mortality after transcatheter aortic valve implantation. Am J Cardiol 114:1447-1455. https://doi.org/10.1016/j. amjcard.2014.07.081

19. Redwood S (2020) The percutAneous Coronary inTervention prIor to trans-catheter aortic VAlve implantaTION, presented at PCR Valves e-Course, 22 November 2020, available at https://www. pcronline.com/Cases-resources-images/Resources/Course-videosslides/2020/PCR-Valves-e-Course-Hotline-I, last accessed on 1205-2021

Publisher's note Springer Nature remains neutral with regard to jurisdictional claims in published maps and institutional affiliations. 